CRITICA, Revista Hispanoamericana de Filosofia

Vol. XXIV, No. 72 (diciembre 1992): 23-46

\title{
SEMÁNTICA TARSKIANA, LENGUAJE NATURAL Y ONTOLOGÍA
}

Alberto Moretti

Universidad de Buenos Aires CONICET

En este artículo se presentan las dos variantes principales de la semántica actual, la cual hace del concepto de condiciones veritativas de una oración la clave para desarrollar las ideas de significado y comprensión. A la luz de una consideración previa acerca del tipo de objetivo teórico perseguido en estos intentos, se examinan algunas críticas y se sostiene que no afectan esencialmente el programa. Se distingue entre las versiones al modo de Davidson de aquellas que siguen ideas de Montague y se sostiene que, cuando el fin teórico primario es explicar la comprensión lingüística, hay razones para dar preferencia a las variantes del primer tipo, y que también las hay cuando se busque una semántica apropiada para caracterizar posiciones ontológicas dependientes de restricciones epistémicas; aunque las preferencias se invierten si la ontología que se quiere expresar va a formularse prescindiendo de consideraciones gnoseológicas.

I

Los sistemas de lógica se desarrollan, en tanto sistemas, describiendo la sintaxis de un lenguaje formal y un conjunto de reglas de transformación aplicables a sus expresiones; y son de lógica cuando se les adjunta una estructura semántica, precisamente 
definida, que involuci a alguna noción de consecuencia lógica. Es comprensible que los conocedores de estos sistemas, al ser enfrentados con el problema de describir y/o explicar la significatividad en los lenguajes naturales, traten de aprovechar su peculiar entrenamiento. Estas personas - los lógicos- tienen, a veces, otro motivo para pretender aplicar sus técnicas de transformación e interpretación al lenguaje natural, un interés menos relacionado con la teoría del lenguaje y más próximo a la gnoseología o a la ontología general. En las obras de Frege, Russell, Carnap, Quine (entre otros), se encuentran ejemplos y razones para la aplicación, en este segundo sentido, de la lógica al lenguaje común. ${ }^{1}$

Dos propósitos, entonces, pretenden justificar el empleo de la "semántica de los lógicos" para estudiar un lenguaje natural. En primer lugar (A), contribuir a explicar aspectos centrales de nuestro comportamiento lingüístico o de nuestro lenguaje, en particular la idea de significación o la de comprensión lingüística. Conviene hacer aquí alguna distinción entre $A_{1}$, predecir o explicar la conducta verbal, y $A_{2}$, explicar la comprensión del hablante, pudiendo con esto referirse al modo como le es posible a un intérprete cualquiera caracterizar la comprensión que le atribuye al hablante o bien a las características de la comprensión que el hablante posee o que se atribuye a sí mismo. Ambos objetivos difieren claramente de $A_{3}$ : especificar formalmente las relaciones de significación que vinculan entre sí a los símbolos que intervienen en la comunicación verbal. ${ }^{2}$

1 Véanse, por ejemplo, el $§ 62$ de los Grundlagen (1884) de Frege y el prefacio de su Begriffsschrift (1879); las conferencias de Russell (1918) sobre el atomismo lógico; los \$§ 95-99 del Aufbau (1928) de Carnap; los $\S \S 33,47$ de Word and Object (1960) de Quine.

2 Hay un proyecto conexo que, para muchos, suena más estimulante: comprender - como distinto de explicar y predecir-, la comprensión lingüística (huir del externalista Hempel hacia el hondo Dilthey), cfr. $\mathrm{H}$. Schneider, "Explanation and Understanding in the Theory of Language", en Baüerle et al. (comp.), Semantics from Different Points of View, Springer, 
En segundo lugar (B), discriminar las categorías básicas de la más clara y simple teoría (o teorías) acerca del mundo que en nuestro lenguaje se ofrece(n) en un momento dado. No es obligado que sean éstas las categorías básicas de nuestras mejores teorias científicas, basta con que, prudentemente, lo sean de aquellas representaciones de la realidad que por motivos más o menos extraños nos parezcan satisfactorias. Sensu lato, este objetivo coincide con el intento de procurar esquemas que correlacionen expresiones lingüísticas con elementos de la realidad de una manera que sea tan independiente como resulte posible de las variaciones entre hablantes y contextos de emisión (con lo cual se aproxima a la forma de ciertas soluciones del problema planteado por $A_{3}$ ).

Cuando el objetivo es de tipo $A$, una justificación para aplicar las técnicas lógicas al lenguaje natural se obtiene a partir de la idea, frecuentemente aceptada, de que parte principal del análisis del lenguaje y la comprensión linguística radica en la determinación de las relaciones entre significados lingüísticos (la estructura semántica). Pero, continúa el argumento, son las oraciones y sus relaciones el aspecto de más directo acceso del funcionamiento del lenguaje y la comunicación; los significados forman parte, si acaso, del aparato té́rico explicativo. Y las relaciones más importantes entre oraciones son las relaciones inferenciales (digamos, su estructura lógica), que pueden verse como restricciones a la distribución de condiciones veritativas én conjuntos de oraciones. Por ende, se concluye, resulta adecuado intentar satisfacer A mediante la aclaración de la estructura lógica del lenguaje. Desde esta perspectiva, la tarea de una teoría semántica será explicar o caracterizar, mediante un conjunto de especificaciones que sea finitamente determinable y empíricamente revisable, lo siguiente: a) los significados de las oraciones; b) los significados de las expresiones no oracionales

Berlín, 1979. Pero no se busca aqư una nómina exhaustiva sino una lista parcial que ayude a ubicar algunas influyentes empresas téricas. 
que componen oraciones; c) las relaciones entre a) y b); d) las relaciones intra a) —con la implicación como paradigma-; e) las relaciones intra b) -paradigma: la inclusión. Con aquel enfoque y estos paradigmas, el modelo de tal teoría se buscará en la semática tarskiana que, básicamente, consiste en la caracterización del predicado veritativo adecuado al lenguaje objeto.

Otra consideración, menos ligada a la tendencia expansionista de los lógicos, también conduce - buscando satisfacer A - cerca de la semántica formal. Supóngase que el rasgo fundamental del comportamiento lingüístico que se procura aclarar sea el fenómeno de la comprensión o interpretación lingüística, sin prejuzgar sobre el papel que en esta tarea cabe a la noción de significado y sus parientes. Desde un punto de vista filosófico, lo que entonces interesa no es interpretar un lenguaje particular sino entender qué es, en general, la comprensión de (y en) un lenguaje particular; esto es, lo que filosóficamente importa es discutir cuál sea la forma general de una teoría de la comprensión lingüística para cada lenguaje particular. ${ }^{3} \mathrm{La}$ idea de significado (y de nexo entre significados), que a veces parece central en la investigación, es sólo un medio para llevarla adelante. Pero se trata de un concepto problemático, sobrecargado de usos, cuyo nuevo empleo convendría demorar. Ahora es natural —en ciertos ámbitos- explorar las posibilidades de una aproximación que proponga que la teoría, básicamente, tiene que referirse a cada oración del lenguaje en estudio y aparearla con lo que preteóricamente podría pasar como su significado; el que estará dado, seguramente, en

3 Veremos que cuando esto se hace siguiendo las líneas davidsonianas se corre el riesgo de conseguir solamente entender cómo conviene diseñar, para cada uno de los miembros de un amplio conjunto de lenguajes naturales, una teoría capaz de representar lo que llamaríamos intuitivamente sus nexos de significaciones -algo análogo a la estructura semántica aludida más arriba- sin por eso alcanzar a dar cuenta del fenómeno de la comprensión. 
algún metalenguaje. Tal asociación deberá hacerse usando la menor cantidad posible de expresiones semánticas y viendo que las usadas sean de las menos problemáticas, es decir, de aquellas que en el metalenguaje encuentran menos dificultades de interpretación; los candidatos, otra vez, son la verdad y los vínculos inferenciales. Se tratará, especialmente, de evitar los giros intensionales cuyo esquema de comprensión es parte del problema general que debe resolverse. En tales condiciones el metalenguaje más tentador resulta ser un lenguaje extensional de primer orden. Pocos pasos distan, desde aquí, hasta las ideas de Davidson.

\section{II}

Se intenta comprender lo expresado lingüísticamente por alguien y, con eso, comprender algo acerca del sujeto que se ha expresado. La descripción de más bajo nivel de un fenómeno de la clase que deberá estudiarse será algo como 'Esa mujer (sea $H$ ) emitió los sonidos tales y cuales'. La primera hipótesis habrá de ser: $H$ emitió una expresión linguíística, y se privilegiarán los casos de emisiones favorables a la hipótesis más específica: $H$ emitió la oración $S$. Dado que el intérprete (digamos $I$ ), en caso de que expresara su intelección de $S$, lo haría en su propio lenguaje -cuya sintaxis le es conocida-continuará mediante una conjetura acerca de la forma de $S$ elaborada sobre la base de sus conocimientos gramaticales. Todas las hipótesis sintácticas que $I$ haga sobre expresiones de $H$ determinarán una teoría sintáctica sobre el lenguaje de $H$ (llamémoslo $\mathfrak{L}$ ) cuya validez empírica dependerá de que la teoría semántica (sobre $\mathfrak{L}$ ) que la utilice resulte empíricamente justificable. De modo que no es esencial que $I$ posea una teoría sintáctica independiente o previa a la construcción de la teoría semántica para $\mathfrak{L}$, y si la poseyese, de todos modos su justificación no será independiente de la de la teoría semántica que la requiera. ${ }^{4}$

4 Naturalmente, si $I$ conociese una gramática para $\mathfrak{L}$ que tuviese alta 
Tenemos, hasta aqui, que $H$ emitió la oración $S$, ahora bien, ¿qué tipo de acto de habla realizó $H$ ?, ¿qué contenido semántico tuvo ese acto? El intérprete $I$ usará algún barrunto teórico sobre fuerzas ilocucionarias para conjeturar, por ejemplo, que $H$ afirmó $D$, siendo $D$ una oración declarativa de $\mathfrak{L}$ que, presuntamente, exhibe el contenido de $S$. Resta interpretar $D$, darle significado, a fin de esclarecer ese contenido. La teorfa que ahora se use debera: i) dar significado a $D$, y también, ii) producir consecuencias observacionales, o al menos aumentar, respecto de las consecuencias observacionales que pudieran derivar de meras conjeturas sintácticas y pragmáticas, los tipos de consecuencias observacionales disponibles.

El primer objetivo podría alcanzarse mediante teoremas de la forma 'El significado de $D$ es (la entidad) $\alpha$ ' o de la forma ' $D$ significa que $p$ 'donde ' $D$ ' es sustituible por nombres de oraciones de $\mathcal{L}$ y ' $p$ ' lo es por oraciones del metalenguaje. Pero la primera variante es poco sutil: reifica significados sin apoyo argumental. Y la segunda variante acude a contextos intensionales de inteligibilidad dudosa en el metalenguaje. Donald Davidson propuso que la forma conveniente de dar significado a $D$ tiene su clave en la generación de un teorema de la forma ' $D$ es verdadera si y sólo si $p^{\prime}{ }^{5}$ Con esto no sólo se superan las dos dificultades señaladas sino que, además, se cuenta con consecuencias de obtención relativamente fácil en los casos en que pueda resultar apropiada una oración $p$ de estructura sintáctica semejante a la de la oración $D$, consecuencias que

confirmación empírica (e independiente), entonces el que la sintaxis conveniente a sus conjeturas semánticas fuese lo más cercana posible a la provista por esa gramática será un elemento de juicio a su favor, y actuará en su contra un alejamiento pronunciado entre ambas. Si en ese caso la semántica elaborada pudiese también sugerir hipótesis sintácticas predictivamente exitosas, I tendría derecho a sentirse interpretativamente muy seguro.

5 'Teorema' sugiere implicación logica a partir de axiomas. Tal pretensión es desmedida. 
también serán de relativamente fácil verificación cuando ocurra que $p$ sea cuasi observacional y pueda entonces contarse con pruebas independientes de que $H$ sostiene $D$. Por lo demás, Tarski ha enseñado de qué modo generar este tipo de teoremas. Llegado a este punto, sobre la base de que $H$ afirmó $D$ y $D$ es verdadera si y sólo si $p$, el intérprete puede, por un lado, interpretar la emisión de $S: H$ afirmó que $p$, y por otro lado, atribuir una creencia a $H: H$ cree que $p$. De esta manera, entonces, el intérprete alcanza a comprender —en buena medida- el contenido de la emisión lingüística de $H$ (el significado de $D$ ), y también consigue comprender algo acerca del emisor que contribuye a explicar esa emisión suya (i.e. cierto estado mental de $H$ ).

En realidad, las últimas conclusiones dependen de que el análisis ofrecido para $S$ pueda integrarse en una teoría que provea análisis para un número suficientemente amplio de oraciones de $\mathfrak{L}$. $^{6}$ Sólo entonces la atribución de actitudes proposicionales a los hablantes de $\mathfrak{L}$ alcanza la magnitud y variedad adecuadas como para constituir una base empírica que confirme la teoría y, así, de la interpretación de $D$, interpretación que, ahora se ve, no queda confinada al bicondicional tarskiano correspondiente?

6 Este holismo rampante no significa, como algunos han creído, que la teorfa afirme la desmesurada tesis de que no se es competente en el manejo de una expresión si no se llegó a conocer el significado de las infinitas expresiones de las que puede formar parte (al cabo, el significado de todas las posibles expresiones del lenguaje). Admitir que la teorfa propuesta debe ser compatible con una explicación sensata de la aprendibilidad del lenguaje y de la comunicación exitosa no está en pugna con una teorfa holista que no necesita atribuir al hablante competente un conocimiento completo del significado de ninguna expresión, y que tampoco implica sostener que todas las relaciones que una expresión tiene con todas las demás contribuyan con igual importancia a la determinación de su función semántica.

7 En M. Platts, Ways of Meaning, Routledge \& Kegan Paul, Londres, 1979 y en W.G. Lycan, Logical Form in Natural Language, MIT, Cambridge (Mass.), 1984, se encuentran excelentes exposiciones crfticas y desarrollos de la teoría semántica de corte davidsoniano. Los textos liminares del 
La forma que Davidson pretende para las teorías semánticas de los lenguajes naturales permite a éstas, frente a cada $\mathfrak{L}$ :

1) dar el significado de (interpretar) una cantidad potencialmente infinita de oraciones de $\mathcal{L}$;

2) mostrar cómo el significado de una oración de $\mathfrak{L}$ es función del significado de sus componentes y del modo de composición generador;

3) prescindir de los significados como entidades;

4) explicar ciertas relaciones de implicación entre oraciones;

5) atribuir actitudes proposicionales a los hablantes de $\mathfrak{L}$ y, con eso (a veces junto con 4), adquirir carácter empírico;

6) caracterizar un conjunto de creencias (deseos, dudas, etc.) básicas de los hablantes de $\mathfrak{L}$;

7) poseer carácter interpretativo, en el sentido de que el conocimiento de la teoría basta para entender o ser capaz de usar $\mathfrak{L}$ competentemente;

8) representar el conocimiento lingüístico implícito del hablante de $\mathfrak{L}$ y hacerlo de un modo que resulte accesible al propio hablante. Para lograr esto último se requiere que el poder expresivo de la metateoría se mantenga tan cerca del aparato conceptual del lenguaje $\mathfrak{L}$ como sea posible.

En el efecto conjunto de 7 y 8 parece residir el núcleo de lo que esta teoría puede ofrecer respecto del objetivo de entender en qué consiste comprender $\mathfrak{L}$. Sin embargo, lo que queda aclarado de modo directo es la posibilidad de que un intérprete que posee cierto lenguaje $\mathfrak{L}^{\prime}$ (que, en general, será distinto de $\mathscr{L})$ comprenda a un hablante de $\mathcal{L}$. Esto es, la teoría, en primer término, es una representación que el intérprete tiene acerca de

propio Davidson se recogen en su Inquiries into Truth and Interpretation, Clarendon, Oxford, 1984. 
$\mathfrak{L}$ y que estará avalada por el hecho de que le permita comunicarse con el hablante. $\mathrm{Si}$, por su parte, el hablante tuviese alguna representación de $\mathfrak{L}$ que explicara su competencia, la estructura de esta representación ¿estaría adecuadamente captada por una teoría de este estilo? Si el hablante típico de $\mathfrak{L}$ fuese un intérprete davidsoniano de sí mismo, entonces aclarar cómo es posible la comprensión de $\mathfrak{L}$ por parte de un intérprete radical también iluminará la comprensión que el propio hablante tiene de sí mismo en tanto hablante de $\mathfrak{L}$. Pero, obsérvese en seguida, este peculiar intérprete carece de un metalenguaje previo y suficiente. Probablemente el mejor fundamento para sostener que la naturaleza de esa autocomprensión es davidsoniana - esto es, que el hablante llega a entender y adquirir su lenguaje construyendo ex parvo un modelo tarskianodebe buscarse en la tesis de que el comportamiento lingüístico está constituido enteramente por la práctica social. Según esto, no será correcto pretender que haya algo en los contenidos semánticos que no provenga de, o no se manifieste en, el intercambio comunicacional.

\section{III}

Desde el campo de los lingüistas —i.e.: aquellos que se plantean objetivos de la clase A de la parte I- se han señalado variadas críticas a las teorías semánticas del tipo de condiciones veritativas (TCV). Se objeta por ejemplo que haya fenómenos semánticos fuera del alcance de una TCV. Es el caso de las oraciones no declarativas cuyo significado - se alega- no puede depender de condiciones veritativas ya que no son oraciones verdaderas o falsas. La crítica prosigue desestimando el análisis de estos enunciados en términos de verbos realizativos, en razón de la necesidad de distinguir entre significado y fuerza ilocucionaria o, siguiendo a Austin, privando de verdad o falsedad también a las oraciones realizativas. Y la introducción de condiciones de cumplimiento (para imperativos) o de 
posibilidad de respuesta (para interrogativas) se ve como un reconocimiento de malsana insuficiencia del análisis de condiciones veritativas. ${ }^{8} \mathrm{La}$ impotencia de este análisis, se ha dicho, también, se aprecia al confrontarlo con el vasto fenómeno de la indexicalidad y con el tan manido tema de las modalidades y las actitudes proposicionales. ${ }^{9}$

También se observa, contra una TCV, que forma parte de la competencia semántica del hablante de un lenguaje natural el comprender que muchas oraciones declarativas carecen de condiciones de verdad cuando no se cumplen ciertas condiciones presupuestas. $Y$ hasta se agrega que aquella competencia incluye, para toda oración natural, el conocimiento de los tipos de actos de habla que pueden realizarse con ella y/o de las condiciones para realizarlos exitosamente y para su uso metafórico, en suma, el conocimiento de las condiciones de su uso apropiado. 10

Algunos formulan fuertes críticas a las TCV por no proveer - presuntamente — ningún indicio acerca de cómo es posible el aprendizaje normal del lenguaje natural, esto es, la adquisición del conocimiento tácito del hablante que la teoría debería representar. ${ }^{11}$

${ }^{8}$ El origen de esta crítica es también el origen de la semántica de los actos de habla, con Austin y Grice desde fines de la década del cincuenta. Cfr. S. Schiffer, Meaning, U.P., Oxford, 1972.

9 Cfr. T. Burge, "Demonstrative Constructions, Reference and Truth", J. of Ph., no. 71, 1974; S. Weinstein, "Truth and Demonstratives", Noûs, no. 8, 1974; J. Wallace, "On the frame of reference", en Davidson y Harman (comps.), Semantics of Natural Language, Reidel, Dordrecht, 1972; W. Sellars, "Language as Thought and as Communication", Ph. \& Ph. Research, no. 29, 1969; Lepore y Loewer, "Absolute Truth Theories for Modal Languages as Theories of Interpretation", Critica, no. 21, 1989.

${ }^{10}$ Cfr. D. Wilson, Presupposition and Non-Truth-Conditional Semantics, Academic Press, Londres, 1975.

${ }^{11}$ Es un tópico que el enfoque chomskiano de la teoría del lenguaje pone énfasis en este problems. 
Varias de estas objeciones se diluyen respecto de la versión de TCV bosquejada en la parte anterior, sobre todo cuando se la vincula con los objetos de tipo A más modestos aludidos en la parte I. Y no es éste el lugar para tratarlas con algún pormenor. ${ }^{12}$ Sin embargo será útil una observación general. Aun respecto a una meta ambiciosa, como la de dar la explicación fundamental del fenómeno de la significatividad lingüística, la fuerza de esas críticas dependería de que las TCV pretendieran explicar completamente todo fenómeno vinculado con el lenguaje. No siendo éste el propósito (y por qué razón, más que el deseo de que el mundo sea simple, cabría abrigar el propósito de explicar todo el lenguaje mediante un solo concepto básico), las críticas acerca de la incapacidad de una TCV para aclarar ciertos fenómenos resultarían graves sólo si estuviesen acompañadas de algún argumento para mostrar que sin explicar aquellos fenómenos huidizos tampoco puede darse una correcta explicación de los demás o, más aún, que en esas condiciones ni siquiera es posible establecer la forma de una explicación adecuada de éstos. ${ }^{13}$ A la ausencia de tal argumento, la defensa de las TCV como explicación de los aspectos fundamentales del significado lingüístico puede sumar razones en favor de la tesis de que la referencia al mundo extralingüístico -y, en general, extramental- es la característica esencial o constitutiva del lenguaje humano. Esto es, defender la idea de que la comunicación procurada por el lenguaje se elabora alrededor de la necesidad de controlar o tener en cuenta (contar con, atender) el medio externo, las cosas y sucesos en general y

12 Para intentos más o menos davidsonianos de lidiar con estos problemas, véanse Lycan, op. cit.; y Lycan y Boer, Knowing Who, MIT, Cambridge (Mass.), 1986.

13 Las TCV de teoría de modelos — presentadas en la parte III- parecen más cómodas ante estos desafíos ya que exhiben una impresionante variedad de "soluciones" de refinado tecnicismo. Véase, por ejemplo, Gabbay y Guenthner (comps.), Handbook of Philosophical Logic, Reidel, Dordrecht, 1989, vol. IV. 
los humanos en particular. Y que entonces, el mayor valor para la supervivencia atribuible al lenguaje (dicho esto en vena ya no trascendental sino naturalista) radica en sus propiedades referenciales. ${ }^{14}$ Sosteniendo así que las nociones de referencia y verdad (o sólo esta última) son la clave para explicar el éxito biológico de la actividad lingüística humana o, mejor quizá, la tendencia al éxito de ciertos estados mentales de los hablantes (crencias, intenciones) y el fenómeno de la transmisión de conocimiento entre los hablantes.

Otros autores han discutido la idea de hacer del concepto de verdad la clave para el esclarecimiento del significado de un modo más directo, no sólo mediante la evaluación de su alcance explicativo. Strawson, por ejemplo, sostuvo que la TCV debe dar alguna explicación de ese concepto y que, al hacerlo de modo razunable tiene que advertirse su dependencia de la noción de intención comunicativa, concepto que de esta manera pasa a ocupar la función teórica central. ${ }^{15}$ Dummett también ha argumentado que no son las condiciones veritativas - las que, prima facie, no son relativas a la capacidad epistémica del sujeto- sino las condiciones de asertabilidad las que deben considerarse básicas para entender el significado. ${ }^{16}$ Hintikka, por su parte, ha criticado el principio de composicionalidad,

14 Un argumento de esta especie se expone y se critica, respectivamente, en H. Putnam, Meaning and the Moral Sciences, Routledge \& Kegan Paul, Londres, 1978; y M. Devitt, Realism and Truth, Blackwell, Oxford, 1984.

15 Véase uno de sus primeros trabajos, "Meaning and Truth", U.P., Oxford, 1969, reimpreso en P. Strawson, Logico-Linguistic Papers, Methuen, Londres, 1971.

16 Cfr., entre otros, M. Dummett, "What is a Theory of Meaning? (II)", en Evans y McDowell (comps.), Truth and Meaning, Clarendon, Oxford, 1976; y C. Wright, Realism, Meaning and Truth, Blackwell, Oxford, 1987. La posicion de Dummett reanima, desde otro ángulo, la cuestion suscitada entre los lingüistas acerca de si deberfan considerar su trabajo como un capítulo de la matemática o de la psicologla cognitiva; cfr. B. Partee, "Semantics-Mathematics or Psychology?", en Baüerle et al. (comps.), Semantics from Different Points of View, Springer, Berlín, 1979. 
sobre el que descansa la TCV, debido a que piensa que existen fenómenos semánticos de relieve que muestran la incorrección de dicho presupuesto. ${ }^{17}$ Aquí sólo menciono estas objeciones, que creo filosóficamente más interesantes, con el único y sibilino comentario de que no me parecen decisivas.

\section{IV}

El objetivo inmediato de una teoría semántica del tipo TCV consiste en especificar un significado para cada oración del lenguaje en estudio, pero haciéndolo mediante la determinación de la manera como ese significado se construye a partir de las contribuciones semánticas de las expresiones suboracionales; esto es, mostrándolo como una función del valor semántico de sus componentes sintácticos. Para una TCV davidsoniana el valor semántico de las expresiones básicas es su valor referencial y la referencia alude siempre a extensiones en el mundo real. Desde esta perspectiva, habiéndose establecido cuáles son los cuantificadores y conectivos extensionales del lenguaje objeto, el núcleo formal de la teoría se completa formulando reglas semánticas apropiadas para definir su uso (ésta no es, por cierto, una secuencia temporal de actividades independientes).

Es evidente que para realizar la tarea programada por una TCV se necesita alguna idea acerca de la composición de la realidad, por lo menos la implícita en lo que pase por conocimiento para el constructor de la teoría, y quizá precisamente ésta cuando el propósito sea la interpretación (A de la parte I). Claro que si el objetivo no fuera dar cuenta de un lenguaje

17 Cfr. J. Hintikka, "A Counterexample to Tarski-type Truth Definitions as Applied to Natural Languages", Philosophia, no. 5, 1975; también J. Hintikka, "On the Developmental of the Model-theoretic Viewpoint in Logical Theory", Synthese, no. 77, 1988. Asimismo, S. Schiffer, "Compositional Semantics and Language Understanding", en Grandy y Warnes (comps.), Philosophical Grounds of Rationality, Clarendon, Oxford, 1986. 
natural procurando especificar lo que el hablante entiende por sus emisiones $\left(A_{2}\right.$ de la parte $\left.I\right)$, sino - B - reformular el conocimiento común (cotidiano y científico) del modo más claro y sistemático o, en todo caso, encontrar las causas que expliquen las emisiones de los hablantes $\left(A_{1}\right)$, se preferirá poner a prueba aquella cosmovisión que constituya la mejor conjetura sobre las categorías elementales de entidades y procesos del mundo, dejando en segundo plano el respeto por las apariencias sintáctico-semánticas del lenguaje natural sobre el que se opere. Vimos también que cabe desentenderse de estas cuestiones más o menos ontológicas y buscar solamente una representación cómoda y eficiente de las conexiones entre significados, sin pretensiones descriptivas de la realidad que vayan más allá de la sistematización del comportamiento lingüístico, vale decir, la previsión de emisiones y el registro de intuiciones sintáctico-semánticas.

La manera davidsoniana de construir TCV busca apoyarse en la representación de la realidad implícita en el lenguaje común. Esta restricción -que tiende a lograr $\mathrm{A}_{2}$ - corre pareja con su idea quineana de que la base empírica de una teoría semántica de $\mathfrak{L}$ es, a la vez, la de una teoría sobre las actitudes proposicionales (las creencias, en particular) de los hablantes de $\mathcal{L}$. Pero la puerta que Davidson entreabrió para el paso quedo de la semántica de los lógicos, fue rápida y escandalosamente atravesada. Las teorías de condiciones veritativas a la manera de Montague autorizan el uso de toda la teoría de conjuntos que haga falta con el fin de representar los sucesos y entidades del mundo de manera que: 1) estos representantes de la teoría de conjuntos puedan utilizarse como objetos directamente referidos o significados por las expresiones y construcciones habitualmente reconocidas en un lenguaje natural, proveyendo de valor semántico a todas ellas; 2) puedan respetarse las intuiciones más arraigadas acerca de la forma sintáctica de las oraciones naturales y así evitar la dicotomía entre forma gra- 
matical superficial y forma lógica (o gramatical) profunda; ${ }^{18}$ 3) se logre reproducir una cantidad significativa de intuiciones semánticas básicas. Consiguientemente, la estrategia que deberá utilizarse consistirá en generalizar la caracterización tarskiana de verdad, relativizándola respecto de estructuras de la teoría de conjuntos llamadas modelos, al mismo tiempo que se desarrolla una minuciosa sintaxis "natural".

El método canónico para obtener una semántica de este tipo (semántica de modelos) comienza con la definición de un lenguaje formal $\mathfrak{F}$ con operadores intensionales y distinción de tipos, al que se le asocia una semántica en términos de modelos. Luego se asigna un tipo semántico a cada categoría sintáctica reconocida en el lenguaje natural $\mathfrak{L}$; se traducen a $\mathfrak{F}$ las expresiones básicas de $\mathfrak{L}$ y finalmente se asigna, a cada regla sintáctica atribuida a $\mathfrak{L}$, una regla de traducción al lenguaje $\mathcal{F}$. La traducción a $\mathfrak{F}$ obrará como método puramente heurístico para la adscripción de denotaciones y sentidos a todas las expresiones de $\mathfrak{L}$; es decir, no se pretende que las expresiones de $\mathcal{F}$ revelen la forma sintáctica profunda de las frases de $\mathcal{L}$. La definición de verdad en un modelo, para $\mathcal{F}$, permitirá entonces asignar condiciones veritativas a todas las oraciones de $\mathfrak{L}^{19}$

18 Por razones ontológicas (también gnoseológicas) Russell puso énfasis en la distinción (aprendida de Frege y Bradley) entre forma lógica y forma gramatical; hasta cierto punto, los esfuerzos de los lógicos venidos a lingüistas procuran confundir (por mor de la ciencia del lenguaje natural) lo que el viejo escéptico trató de separar. La lógica se roza con la ontología y con la lingüística; en los últimos tiempos los lógicos han preferido notoriamente estudiar la relación con el lenguaje ordinario relegando el trato con el resto del mundo; no es descabellado preguntarse cuánto ha influido en este vuelco la diferencia entre los sueldos ofrecidos por los departamentos de filosofía y los departamentos de investigación lingüística e informática de las universidades $y / o$ industrias financieramente poderosas.

19 Textos básicos son D. Lewis, "General Semantics", en Davidson y Harman (comps.), Semantics of Natural Languages, Reidel, Dordrecht, 1972; y los trabajos de R. Montague reunidos en Formal Philosophy (compilados por Thomason), Yale U.P., N. Haven, 1974. Un acercamiento pau- 
La principal ventaja de las teorías semánticas construidas de este modo, reside en que permiten ofrecer definiciones matemáticamente aceptables de conceptos semánticos fundamentales como los de implicación, validez y equivalencia lógica. Estas definiciones dan lugar a la demostración rigurosa de metateoremas que hacen interesante la teoría desde el punto de vista matemático. Los conceptos semánticos y sus relaciones quedan así caracterizados de manera muy clara y distinta. ${ }^{20}$

\section{V}

La evaluación de una teoría como la de Montague se hace con referencia a un conjunto de intuiciones semánticas del hablante, tales como ciertos juicios más o menos comunes acerca de ambigüedad, implicación, equivalencia, sinonimia. Uno de los éxitos de estas teorías, por ejemplo la clásica PTQ, ha sido el poder asignar de modo directo las interpretaciones que distinguen a 'Todo hombre calla o habla' de 'Todo hombre calla' o 'Todo hombre habla'; PTQ también es capaz de describir la ambigüedad que exhiben oraciones como 'Andrés cree que un espía se acerca' y puede evitar los problemas inferenciales planteados por muchos contextos oblicuos. Uno de sus deméritos importantes ha sido su incapacidad para justificar el fallo inferencial en contextos de creencia cuando hay sustitución de oraciones logicamente equivalentes. Aun otras teorías posteriores, que mejoran PTQ en este último sentido, no logran superar todas las dificultades que plantean estos contextos. ${ }^{21}$ Pero hay otra clase de incomodidades.

sado a estas teorías se encuentra en Dowty, Wall y Peters, Introduction to Montague Semantics, Reidel, Dordrecht, 1981.

20 Para una apoteosis de este enfoque puede verse la compilación de Gabbay y Guenthner citada en la n. 13.

21 Cfr. Lewis, “General Semantics”, op. cit., y también M.J. Cresswell, Structured Meanings: The Semantics of Propositional Attitudes, MIT Press, Cambridge (Mass.), 1985. 
¿Cuál es el papel que en estas teorías desempeña el aparato de la teoría de conjuntos del metalenguaje? O bien es sólo un método para dar una descripción matemáticamente precisa de las relaciones intralingüísticas que se fundamentan en el nexo entre lenguaje y realidad; o bien debe adscribírsele también la pretensión de representar la estructura del mundo con que se vincula al lenguaje.

En el segundo caso, un conjunto como el de los mundos posibles el conjunto $W$ que es componente obligado de los modelos- es un conjunto de entidades con algún género de realidad, aunque no siempre sea el ser actual o efectivo. Surgen entonces dos problemas. En primer lugar, el de caracterizar convenientemente ese tipo de entidades (¿por qué $W$ no sería un conjunto de mariposas o de galaxias?) y mostrar su fertilidad en otros campos. Un argumento que dijera que puesto que la teoría explica los juicios habituales acerca de implicaciones y equivalencias, entonces eso ofrece suficiente apoyo para afirmar la realidad de las entidades teóricas que necesita, olvidaría que se requieren mayores restricciones para asignar audaces referencias a los términos teóricos de los enunciados empíricos (de no hacerlo, siempre podrían invocarse duendes serviciales de los que sólo se sabe que cumplen exactamente la función que conviene a la teoría y permanecen ajenos a cualquier otra cosa o proceso). Restricciones tales como requerir que no obliguen a reestructurar gravemente otras teorías o principios generáles de sólido arraigo, y que tengan un papel en la explicación de otro tipo de fenómenos. En este caso, tal vez, fenómenos de índole psíquica o social, por ejemplo: el conocimiento implícito del lenguaje o el proceso de su adquisición, o el de la comunicación entre hablantes. Porque, después de todo, los mundos y los objetos meramente posibles entendidos platónicamente, ¿cómo podrían relacionarse con fenómenos del mundo efectivo? Parece que una interpretación conceptualista sería más adecuada para enfrentar esta demanda; sin embargo, eso podría dificultar la demostrabilidad de los metateoremas que ha- 
cen matemáticamente interesante la teoría. Analizar la jerga de mundos posibles en términos de condicionales contrafácticos también deteriora este aspecto y además compromete el desideratum de no emplear construcciones metalingüísticas de dudosa claridad semántica. ${ }^{22}$

En segundo lugar, según sea esa caracterización se hará difícil justificar la adscripción de creencias al hablante sobre la base de las condiciones veritativas que la teoría señale para las oraciones que él acepte. Es evidente que lo que el hablante dice, el significado de lo que él dice -a diferencia de lo que el teórico encuentre como explicación o expediente sistematizador de las emisiones del hablante- debe estar en relación con sus creencias e intenciones. Atribuir significados de manera realista y no meramente instrumental, es interpretar y, presumiblemente, atribuir "actitudes proposicionales". Si el lenguaje objeto no tiene recursos como para expresar una poderosa teoría de conjuntos ni un concepto de la complejidad que se le conceda al de mundo posible (o si tiene recursos que hacen innecesario el rodeo de la teoría de conjuntos), entonces la metateoría no servirá para comprender lo que los hablantes creen, aunque pudiese emplearse para aclarar cuál es — según el punto de vista del intérprete-la realidad que esos hablantes expresan, sin comprenderla adecuadamente, con las oraciones que aceptan. Por esta razón una teoría de este tipo, que se entendiera de modo realista por la elucidación de alguno de sus primitivos, podría utilizarse para satisfacer el objetivo teórico $B$ de la parte $I,{ }^{23}$ pero no resulta apropiada en general (si es

22 Estas observaciones no implican que la idea de mundo posible y de cognadas sea empíricamente vacua, sino sólo que para alentar su carácter emṕrico se necesita argumentación adicional. Véanse, por ejemplo, Loux (comp.), The Possible and the Actual, Cornell U.P., 1979; D. Lewis, On the Plurality of Worlds, Blackwell, Oxford, 1986; R. Stalnaker, Inquiry, MIT Press, Cambridge (Mass.), 1987.

23 Aunque entonces ipor qué evitar la dicotomía forma gramatical su- 
que va a exceptuarse el caso homofónico) cuando el objetivo buscado sea el indicado en $A$ (parte I).

A este respecto, vale decir, al considerar su eficacia para satisfacer A, también se ha visto ${ }^{24}$ otra desventaja de estas teorías respecto de las del tipo davidsoniano: con estas últimas se conseguiría explicar, vía deducción, hechos semánticos fundamentales como los exhibidos por los bicondicionales tarskianos; las teorías de modelo, en cambio, no logran esas explicaciones a menos que incluyan conjeturas explícitas acerca de las extensiones en el mundo efectivo de predicados y términos singulares; pero cuando lo hacen, todo su aparato de teoría de conjuntos resulta superfluo. Sin embargo, no es cierto que una TCV davidsoniana logre aquellas implicaciones, ${ }^{25}$ parece más cerca de lograrlo debido a que su metalenguaje se mantiene próximo al lenguaje objeto, sin embargo, ésta es la misma desventaja relativa implícita en el párrafo anterior.

Si en cambio se adopta la primera vía de la alternativa concerniente al metalenguaje de teoría de conjuntos y éste se entiende tan sólo como un expediente para sistematizar la trama de significados o, mejor aún (para eludir reificaciones no deseadas), la trama de expresiones significativas, queda entonces nuevamente cortada, aunque de manera más drástica que antes, la conexión entre significatividad y creencias. Pero esto plantea una dificultad relativa a la base empírica de la teoría. En efecto, si no existe la posibilidad de evaluarla según la razonabilidad del sistema de actitudes proposicionales que adjudica a los hablantes, su carácter empírico depende sólo de que pueda contarse con un conjunto previo de intuiciones semánticas acerca de implicaciones y sinonimias. Esto último

perficial/forma lógica? Más aún, en un caso así resulta algo más plausible afirmarla que desecharla.

${ }^{24}$ Cfr. E. Lepore, "What Model Theoretic Semantics cannot Do?, Synthese, no. 54, 1983.

25 Cfr. W.G. Lycan, op. cit., cap. 2, n. 7. 
es accesible cuando el lenguaje objeto es parte del metalenguaje y se sabe que lo es (¿cómo se sabe?). ${ }^{26}$ Cuando esto no ocurre parece muy lejana la posibilidad de constituir una adecuada y suficiente colección de intuiciones semánticas para sustentar la teoría. Sin duda será mucho más difícil que el ya penoso método davidsoniano de recurrir a las oraciones que los hablantes parecen "sostener como verdaderas" y de las cuales cabe conjeturar que son de bajo nivel teórico.

Estas observaciones llevan a concluir que las teorías de modelos no ejemplifican la forma que una teoría semántica ha de tener para proveer un análisis explicativo de la comprensión de un lenguaje natural. $Y$ aclarar este punto es una (si no la) tarea fundamental para la "filosofía del lenguaje". Las versiones davidsonianas de TCV son más aptas para alcanzar esa finalidad; aunque, si existe diferencia cualitativa entre la interpretación radical planteada por Davidson y la autocomprensión del hablante, no es probable que sean útiles o suficientes para describir o explicar este último fenómeno. Por otra parte, en lo que hace a capacidad para predecir la conducta verbal, no parece haber razones de principio en favor de alguno de estos tipos de $\mathrm{TCV}^{27}$ En cuanto a la presencia de conceptos básicos pro-

26 Como ejemplo elemental del tipo de dificultades ímplicitas aun en estos casos favorables, considérese uno de los primeros éxitos de este enfoque. 'Todo perro ladra' y 'Algún perro ladra' tienen la misma forma gramatical: NP + VP; pero el análisis lógico habitual lleva a concluir que sus formas lógicas (sus sintaxis profundas) son muy diferentes entre sf y distintas de su forma superficial. La teorfa PTQ, no obstante, legitima la gramática aparente de estas oraciones y les asigna análisis semánticos paralelos. Según esto 'Todo perro' es sujeto y denota una propiedad de propiedades de individuos, y 'ladra' es predicado y denota una propiedad de individuos. Pero, ino es intuitivo que la VP denota lo dicho acerca de lo nombrado por la NP?, ¿no es ésta una intuición semántica tan valiosa -al menos - como la intuición sintáctica de que aquellas oraciones en efecto tienen la forma que parecen exhibir? Sin embargo, luego del análisis producido por PTQ, el sujeto sintáctico ha pasado a desempeñar la función semántica (intuitiva) de predicado y viceversa.

27 Sin embargo, no es claro que la TCV davidsoniana pueda responder 
blemáticos, las teorías davidsonianas - y no las otras- permiten eliminar algunas nociones del llamado "círculo intensional": aunque en su desarrollo completo recurran a creencias e intenciones, eluden el concepto de significado y sus derivados. $\mathrm{Y}$ es plausible creer que si bien no hay hechos semánticos al margen de lo que se derive del comportamiento público de los hablantes - hechos semánticos recónditos para los que probablemente no haya explicaciones extrasemánticas-, sí existen hechos psíquicos, sociales y físicos parcialmente explicativos de las creencias e intenciones.

Sin embargo, es útil advertir que la teoría davidsoniana es heredera directa de las ideas de Quine acerca de cómo examinar el lenguaje natural cuando se tiene como interés primordial un objetivo de tipo B y no A (tal como lo hicieran ancestros de Quine, como Frege, Russell o Carnap). Da la impresión de que se ha producido un deslizamiento insuficientemente justificado desde el (presuntamente exitoso) análisis lógico del lenguaje motivado por preocupaciones onto-gnoseológicas hacia su estudio mediante procedimientos similares (la "semántica de los lógicos") pero ahora con fines lingüísticos. Pero cuando, más que lo dicho por alguien, lo que importa es entender ese decir o al sujeto que dice, parece razonable pensar que el núcleo de

simultáneamente a las demandas de $A_{1}$ y $A_{2}$ (cfr. la parte I). Considérese, para simplificar, un único bicondicional, ' $D$ es verdadera si y sólo si $p$ '. La expresión 'es verdadera' pertenece al metalenguaje, y para que sea reconocida como el predicado veritativo adecuado al lenguaje objeto, el lado derecho del bicondicional debe dar las condiciones que el térico cree que deben existir para que $D$ sea verdadera. Pero entonces, no es -en general- legítimo atribuir al hablante la creencia de que $p$. Es dudoso que la caridad cognoscitiva, recomendada por Davidson en estas circunstancias, sea un resguardo suficiente. Con lo cual se pierde base empírica y se aleja la posibilidad de cumplir $A_{2}$. Aunque el resultado pueda usarse como parte de un esquema para cumplir $A_{1}$ (predecir emisiones). $\mathrm{Si}$, por otro lado, $p$ no describe el hecho que verificaría $D$, entonces 'es verdadera' no es el predicado veritativo del lenguaje objeto y la teoría resultante tal vez sirva para $A_{2}$ pero dejaría de ser una TCV. Aquí es donde la sombra de M. Dummett regresa. 
una teoría explicativa no sea la semántica formal sino alguna combinación entre las teorías de la mente, la sociedad y la acción humana. ${ }^{28}$

Una observación final. Considérense tres fines por los cuales emprender el estudio de un lenguaje natural: 1) explicar la conducta verbal (de alguna comunidad o individuo); 2) esclarecer la ontología naif ${ }^{29}$ (de alguna comunidad) a través de sus manifestaciones en las estructuras lingüísticas; 3) establecer un lenguaje adecuado para exponer cierta concepción ontológica.

Determinar cuál sea la ontología naif bien puede ser un problema científico. Su solución dependerá —en ese casode la adopción de teorías psicológicas, sociológicas, físicas, lingüísticas y conexas que estarán "autorizadas" por alguna gnoseología que, sin embargo, de ser "naturalizada", recaerá finalmente sobre aquéllas. Parece claro pues, que 1) y 2) están más relacionados entre sí que lo que 2) y 3) lo están.

Por otra parte, la concepción ontológica aludida en 3) puede ser una que aspire - por razones de simplicidad, intuitividad, etc.- a conformarse a (y con) nuestras mejores teorías científicas. Se tratará entonces de una ontología naturalizada que - vía las teorías científicas-mantendrá estrecho vínculo con consideraciones gnoseológicas. Esta referencia a la mejor ciencia, además, separa a 3 ), nítidamente, de 2). Aunque también cabe que sea de otra clase. Una especie de ontología libre (¿desnaturalizada?) que acentúe aspiraciones de coherencia

28 No queda excluido el intento por hacer de la semántica tarskiana el núcleo de alguna de estas teorfas (cfr. H. Field, "Mental Representation", Erkenntnis, no. 13, 1978), pero, sin duda, se descarta que esto pueda considerarse algo obvio.

29 'Ontología' tiene aquí — cuando menos- un sentido afín al que la escolástica daba a "philosophia prima' o al que le atribuyó el siglo XVIII o los autores de "Cegenstandstheories" o Quine. 
lógica y se conecte débilmente con la gnoseología. Distingamos dos requisitos: a) permitir explicar (o al menos predecir) la conducta o la experiencia de los sujetos epistémicos; b) ser cognoscible como tal por parte de los sujetos epistémicos. Una teoría ontológica a la que se le exija a) pero no b), tendrá que postular hechos o realidades que fundamenten la mayoría de las creencias fuertes de los sujetos epistémicos, pero no tendrá que estructurarlas de modo que los sujetos sean capaces de acceder cognoscitivamente a ellas, esto es, capaces de justificar debidamente sus pretensiones de haber accedido a ellas o, siquiera, capaces de argumentar a favor de este acceso de mejor manera que quienes lo hagan respecto de otro tipo de hechos o realidades. Para desafueros así, los recursos adoptados por la semántica de modelos sortean prima facie los reparos que la disminuían frente a su alternativa davidsoniana.

Las construcciones de una ontología de este tipo serán intentos por mostrar cómo es posible que la realidad sea cognoscitivamente inalcanzable por nosotros, más que intentos por determinar cuál pueda ser la ontología-para-nosotros. Algo como la exposición de cuán variables estructuras, procesos y entidades reales pueden estar referidas por nuestras creencias, sin que seamos capaces de elegir entre ellas usando solamente razones que sean independientes de nuestras limitaciones epistémicas. Las teorías de esta clase resultarán productos del pensar descargado del empeño y los recaudos y cautelas propios de quien trata de fijar conocimientos. Justamente — según dicen- lo que Kant juzgaba inútil. Pero éste ya es otro problema. ${ }^{30}$

Recibido: 15 de octubre de 1991

30 La primera versión de este trabajo fue leída, parcialmente, en el XII Congreso Interamericano de Filosofía (julio, 1989). Agradezco el apoyo de la Fundación Antorchas que hizo posible esta versión revisada. 


\section{SUMMARY}

As a first step, this paper presents the two main variants of the contemporary semantical approach which takes truth-conditions as the key-concept to develop the ideas of meaning and understanding. Some criticisms of that approach are examined in light of that approach's theoretical goal. My claim is that the criticisms do not essentially affect the programme. Differences are pointed out between Davidson's and Montague's variants within this programme. My thesis is that when the main theoretical goal is to explain linguistic understanding; there are reasons to prefer the former. There are also reasons for this choice when the goal is to look for a semantics appropriate for characterizing ontological positions that depend upon epistemic constraints. However, Montague's variants are preferable when one tries to state an ontology without taking into account epistemic factors.

[Traducción de Raúl Orayen] 